\title{
Membrane Deformability and Membrane Tension of Single Isolated Mitochondria
}

\author{
Shiqi Wang, ${ }^{1}$ Chunsun Jiang, ${ }^{2}$ Yan Zhang, ${ }^{1}$ Juan Chen, ${ }^{1}$ Bin Wang, ${ }^{2}$ Quan Chen, ${ }^{2}$ and Mian Long ${ }^{1}$ \\ ${ }^{1}$ National Microgravity Laboratory, Center for Biomechanics and Bioengineering, Institute of Mechanics, Chinese Academy \\ of Sciences, Beijing 100190, P.R. China; and ${ }^{2}$ The Laboratory of Apoptosis and Cancer Biology, The National Key Laboratory \\ of Biomembrane and Membrane Biotechnology, Chinese Academy of Sciences, Beijing 100101, P.R. China
}

(Received 7 December 2007; accepted 23 January 2008; published online 4 March 2008)

\begin{abstract}
Mitochondria dynamics is crucial to many biological processes such as mitochondria fusion and fission, which is highly correlated to the mechanics of single mitochondria. However, the mechanobiological coupling of mitochondria has been poorly understood. Here membrane deformability and membrane tension of individual mitochondria isolated from MtDsRed labeled human embryonic T-Rex-293 kidney cells were measured using a micropipette aspiration assay. The results demonstrated that membrane deformation of isolated mitochondria exhibited an elastic transition phase followed by an equilibrium phase, and mitochondrial membrane tension was proportional to the area compressibility. It was also indicated that mitochondrial membrane deformability was significantly affected by physical-chemical factors such as osmotic pressure or $\mathrm{pH}$ value, and was further correlated to mitochondrial functionality in different respiratory states and $\mathrm{Ca}^{2+}$ regulation. These findings provide a new insight into understanding the mechanical regulation of mitochondrial physiology.
\end{abstract}

Keywords-Mechanical properties, Physical-chemical environment, Respiratory state, Membrane permeability transition.

\section{INTRODUCTION}

Mitochondria play a central role in determining cell life and death. Mitochondrial deficiencies accumulating with time are crucial to many pathophysiological processes such as Parkinson's disease, Alzheimer's disease, diabetes mellitus, liver disease, muscle dystrophy, cardiomyopathy, and cancer., ${ }^{3,7,10}$ As an important subcellular compartment in almost all kinds of eukaryotic cells, mitochondria have the structure

Address correspondence to Mian Long, National Microgravity Laboratory, Center for Biomechanics and Bioengineering, Institute of Mechanics, Chinese Academy of Sciences, Beijing 100190, P.R. China. Electronic mail: mlong@imech.ac.cn that contains the outer membrane, the intermembrane space, the inner membrane, and the matrix. The inner membrane is highly folded into cristae, in which there are the complexes of electron transport chain and ATP synthase that control the basic rates of cellular metabolism. In the past decade, mitochondria are mostly considered to serve as fundamental elements in biochemical signaling since current approaches have enabled to examine dynamic functionality and behavior of mitochondria. ${ }^{18}$

To perform their biological functions in electron transfer and respirations, mitochondria are required to form a highly dynamic, continuously-evolved reticulum or network in a cell, not only for the evolution of morphology but also for the manipulation of size distribution and copy number. For example, mitochondria in differentiated cells are often localized to specific cytoplasmic regions rather than randomly distributed. ${ }^{24}$ Mitochondria also proceed a continuous cycle of dynamic fusion and fission in a live cell. During the cycle, mitochondria undergo retrograde and anterograde movements along the microtubule in combination with such motor proteins as myosins, dyneins, or kinesins. ${ }^{9,21,22}$ Mitochondrial movement, fusion and fission are often accompanied with their own active deformation, suggesting that mechanics of mitochondria play an important role in the process. In a reconstructed vesicle of cardiolipin which is the unique negatively charged lipid appearing predominantly within the inner mitochondrial membrane, both apparent area compressibility modulus and lysis tension are found to decrease with increasing cardiolipin content. ${ }^{15}$ To date, however, mechanics of mitochondria and their relevance to biological functionalities have not been well understood.

In the present work, a quantitative in vitro approach was developed to investigate mechanics of isolated mitochondria and their correlation with mitochondrial functionality in respiration. Individual mitochondria 
were isolated from human embryonic T-Rex-293 kidney cell lines labeled with MtDsRed. Membrane deformability and membrane tension, which are important constituent of mitochondrial membrane mechanics, were then measured using a micropipette aspiration assay, a well-developed technique widely used in cell mechanics and rheology. ${ }^{8,14,23}$ Our results indicated that membrane deformation of isolated mitochondria exhibited an elastic transition phase followed by a saturation phase, and their respiratory functionality was coupled to the membrane tension of mitochondria.

\section{MATERIALS AND METHODS}

\section{Reagents}

CCCP (carbonyl cyanide 3-chlorophenylhydrazone) and antimycin were purchased from Sigma Chemical Co. (London, UK). CsA (cyclosporine A) was purchased from Calbiochem (San Diego, USA). All other reagents were from Beijing Chemical Reagents Company (Beijing, China) with the highest purity commercially available.

\section{Cell Transfection, Culture and Mitochondria Isolation}

Human embryonic T-Rex-293 kidney cells (Invitrogen, USA) were transfected by MtDsRed, a plasmid encoding a mitochondrial matrix-targeting protein, using DMRIE-c (Life Science, USA) upon the manufacturer's instructions. Transfected cells were grown up in Dulbecco's modified Eagle medium (DMEM) (Hyclone, Logan, USA) containing 10\% heat-inactivated fetal bovine serum (FBS) (Gibco, Tulsa, USA) and $1 \%$ of penicillin and streptomycin each (Hyclone, Logan, USA). For mitochondria isolation, cells were harvested by $0.25 \%$ trypsin (Hyclone, Logan, USA). The suspension were centrifuged at $1500 \mathrm{rpm}$ for 3 min and washed in PBS $\left(\mathrm{Ca}^{2+}\right.$ - and $\mathrm{Mg}^{2+}$-free). Cell pellets were then resuspended in $150 \mu \mathrm{L}$ of Dounce buffer $\quad(210 \mathrm{mM}$ sucrose $/ 70 \mathrm{mM}$ mannitol $/ 1 \mathrm{mM}$ EGTA/10 mM HEPES, pH 7.4) on ice for $30 \mathrm{~min}$, and the cell suspension was transferred to the Dounce homogenizer embedded on ice. Homogenization was accomplished by $30-40$ vigorous, directly vertical passes of muller B. Resulted homogenate was transferred to an eppendorf tube and the supernatant was collected after being centrifuged at $800 \mathrm{~g}$ for $10 \mathrm{~min}$. Again, the collected supernatant was re-centrifuged at $10,000 \mathrm{~g}$ for $10 \mathrm{~min}$ and the final precipitate (mostly mitochondria) was resuspended in $50 \mu \mathrm{L}$ Dounce buffer on ice.

\section{Electron Microscopy Observation}

Isolated mitochondria as above were fixed with $2.5 \%$ glutaraldehyde for 15 min. After fixation, the samples were washed thoroughly with PBS and fixed in $1 \% \mathrm{Os}_{2} \mathrm{O}_{4}$ for $2 \mathrm{~h}$ at $4{ }^{\circ} \mathrm{C}$. Dehydrated samples were embedded in Spur ${ }^{\mathrm{TM}}$ for $24 \mathrm{~h}$ at $65^{\circ} \mathrm{C}$. After being stained with uranyl acetate and lead citrate, the sections were observed using a transmission electron microscope (TEM) (Tecnai 20, Fei, Hillsboro, USA), and the images were collected by a CCD Camera (Gatan USC1000, USA).

\section{Micropipette Aspiration Assay}

The micropipette aspiration system consists of an inverted microscope with a $100 \times$ oil immersed objective (IX70 Olympus, Tokyo, Japan), a micromanipulator with a resolution of $\sim 0.2 \mu \mathrm{m}$ (MMN-1 Narishige Co. Japan), a suction pressure unit with a resolution of $10 \mu \mathrm{m} \mathrm{H}_{2} \mathrm{O}$ (corresponding to a minimum suction pressure of $0.98 \mathrm{dyn} / \mathrm{cm}^{2}$ or $0.098 \mathrm{pN} / \mu \mathrm{m}^{2}$ ), a CCD camera (WAT-902H Watec, Yamagata-Ken, Japan), and a tape recorder (Panasonic Co.). ${ }^{12,23}$ A typical micropipette microforged to be of an inner diameter of $0.5-1.0 \mu \mathrm{m}$ was mounted onto the micromanipulator. After zeroing the pressure at the open end, the micropipette was driven to be close to the targeted mitochondrion in solution, and a slight suction pressure was applied to trap it before suction pressure was jumped instantaneously to the preset value ranging from 25 to $150 \mathrm{~mm} \mathrm{H}_{2} \mathrm{O}$ ( $c f$. Fig. 2f). Time course of mitochondrion deformation was recorded using a tape recorder at 25 frames per second, and the time dependence of tongue length inside the micropipette, $L$, was obtained ( $c f$. Figs. 2a-e and 3a).

To conduct the mechanical measurements, $1 \mu \mathrm{L}$ of isolated mitochondria in Dounce Buffer was injected into a customer-made chamber containing $1 \mathrm{~mL}$ of physiological buffer (PB) $(250 \mathrm{mM}$ sucrose $/ 3 \mathrm{mM}$ Hepes $/ 0.5 \mathrm{mM} \quad$ EGTA/2 mM $\quad \mathrm{KH}_{2} \mathrm{PO}_{4}, \mathrm{pH}$ 7.4). Membrane deformability of mitochondria was extensively tested in different physical-chemical factors of osmotic pressure and $\mathrm{pH}$ value, as well as in various respiratory states modulated by addition of succinate, ADP, CCCP, antimycin, $\mathrm{Ca}^{2+}$, or CsA. Total 17-30 mitochondria were measured in each case. All measurements were done at room temperature $\left(19-21^{\circ} \mathrm{C}\right)$.

\section{Data Analysis}

Recorded images of mitochondrial deformation at a magnification of 5200x were digitalized and the dimensions were measured off-line at the resolution of $\sim 1$ pixel $(\sim 0.09 \mu \mathrm{m})$. At least duplicate measurements 
were repeated for each dimension of micropipette diameter, intact or aspirated mitochondrion diameter or aspirated mitochondrion tongue to obtain the averaged dimension. Dimensionless tongue length $\left(L_{\mathrm{m}} / R_{\mathrm{p}}\right)$ of mitochondrion was defined as the ratio of maximum length, $L_{\mathrm{m}}$, to radius of micropipette, $R_{\mathrm{p}}$, at the given suction pressure, $\Delta P$. The statistical significance of (or the lack thereof) the difference between the dimensionless tongue length of mitochondrion presented in different physical-chemical and respiratory environments was assessed using the Student t-test.

Membrane surface tension of mitochondrion at $\Delta P$ was calculated by LaPlacian law:

$$
\Delta P=2 T \times\left(1 / R_{\mathrm{p}}-1 / R_{\mathrm{m}}\right),
$$

where $T$ is membrane tension and $R_{\mathrm{m}}$ is the equilibrium radius of mitochondrion. The area strain, $\Theta=\Delta A / A_{0}=\left(A-A_{0}\right) / A_{0}$, where $A$ and $A_{0}$ are the equilibrium area at $\Delta P$ and the original area at $\Delta P=0$, respectively, was calculated using a wellknown formulation, ${ }^{11}$

$$
\Theta=\Delta A / A_{0} \approx\left[\left(R_{\mathrm{p}} / R_{\mathrm{m}}\right)^{2}-\left(R_{\mathrm{p}} / R_{\mathrm{m}}\right)^{3}\right] \times L_{\mathrm{m}} /\left(2 R_{\mathrm{p}}\right)
$$

The area compressibility modulus, $K_{\text {area }}$, of isolated mitochondria was obtained from the slope of surface tension vs. area strain fitted using a straight line.

\section{RESULTS}

\section{Mitochondrial Morphology and Size Distribution}

Isolated mitochondria from transfected T-Rex-293 cells in PB were observed as red vesicles using fluo- rescent microscope (Fig. 1a). In contrast to mitochondria existing in a living cell where individual mitochondrion has an elliptic geometry and tends to form the inter-mitochondrial reticulum via instantaneous fusions and fissions, most of mitochondria in such an in vitro assay presented round geometry and some of them formed small aggregates (doublets, triplets) (Fig. 1a). Ultra-microscopic observations using TEM images demonstrated that isolated mitochondria had intact inner and outer membranes (cf. Fig. 6a). Geometry of isolated mitochondria was also quantified directly from fluorescent images. As exemplified in Fig. 1b, typical size distribution had a bell shape where the peak value of mitochondrial diameter was between 0.5 to $1.5 \mu \mathrm{m}$. Similar size distribution of mitochondria was also found in the medium of $\mathrm{PB} / 10 \mathrm{mM}$ succinate, $\mathrm{PB} / 10 \mathrm{mM}$ succinate/ $0.5 \mathrm{mM}$ ADP, or $\mathrm{PB} / 10 \mathrm{mM}$ succinate $/ 1 \mathrm{mM} \mathrm{Ca}^{2+}$, respectively, where the peak value also ranged from 0.5 to $1.5 \mu \mathrm{m}$ (data not shown). Taken together, theses data indicated that isolated mitochondria presented stable size distribution.

\section{Mechanical Responses of Mitochondria}

A modified micropipette aspiration assay ${ }^{8,12,14,23}$ was used to measure mechanical responses of isolated mitochondrion at a given suction pressure $\Delta P=100$ $\mathrm{mm} \mathrm{H}_{2} \mathrm{O}$. As visualized in Figs. $2 \mathrm{a}-\mathrm{e}$, the tongue of mitochondrion went quickly into the micropipette and the mitochondrial deformation was then slowed down sharply. This was further validated by quantifying the time dependence of tongue length. As exemplified in Fig. 3a, the membrane deformation exhibited a transition phase where $L$ increased with $t$ and a steady phase where $L$ reached equilibrium. The typical maximum
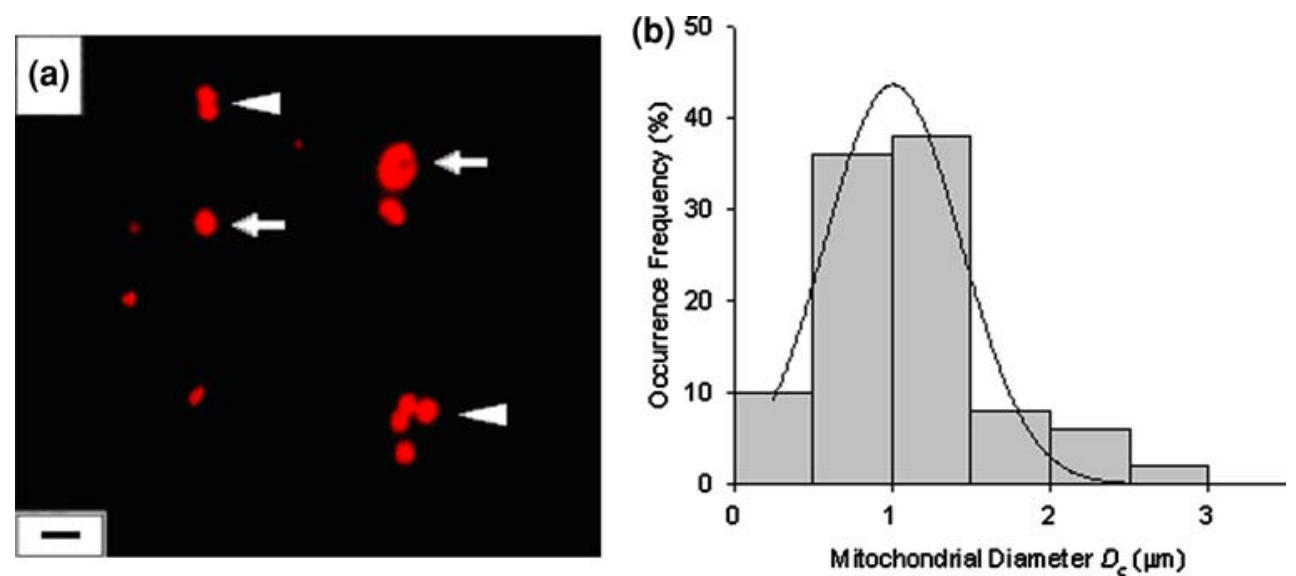

FIGURE 1. Geometry and size distribution of individual mitochondria. (a) Fluorescent images of MtDsRed labeled mitochondria isolated from transfected T-Rex-293 cells were recorded by light microscope. Arrows and arrow heads indicated single mitochondria and mitochondrial aggregates (doublets and triplets), respectively. Bar $=2 \mu \mathrm{m}$. (b) Typical size distribution of isolated mitochondria suspended in PB (line is the trend line.). 



FIGURE 2. A single mitochondrion suspended in PB was aspirated at $\Delta P=100 \mathrm{~mm} \mathrm{H}_{2} \mathrm{O}$. Typical time course of mitochondrial deformation was presented at $t=0$ (a), 120 (b), 240 (c), 360 (d), and $1200 \mathrm{~ms}$ (e). Bar $=2 \mu \mathrm{m}$. Schematic of micropipette aspiration assay was illustrated (f), where $R_{\mathrm{m}}$ and $\boldsymbol{R}_{\mathrm{p}}$ are the radii of mitochondrion and micropipette, respectively, and $L$ is aspirated tongue length.
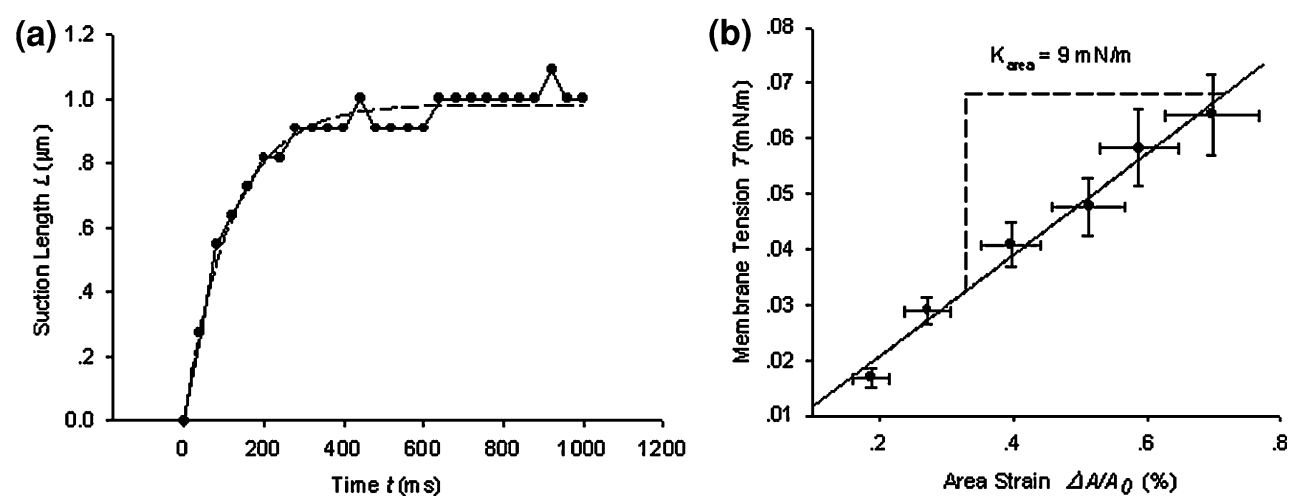

FIGURE 3. (a) Typical time dependence of aspirated tongue length of mitochondria suspended in $P B$ at $\Delta P=100 \mathrm{~mm} \mathrm{H}_{2} \mathrm{O}$. Points are the measured data and dash line is the trend line. (b) Area strain dependence of surface tension for mitochondria suspended in PB at $\Delta P=25-150 \mathrm{~mm} \mathrm{H}_{2} \mathrm{O}$. Data (points) are presented as the mean \pm standard errors $(N=19)$ (solid line is the linear fitted line).

tongue length, $L_{\mathrm{m}}$, amounts to $1.09 \mu \mathrm{m}$ where the average micropipette radius $R_{\mathrm{p}}=0.30 \pm 0.04 \mu \mathrm{m}$. This turned to be the dimensionless suction length $L_{\mathrm{m}} / R_{\mathrm{p}}=0.78 \pm 0.09$.

Membrane tension of isolated mitochondria was further tested by systematically varying suction pressure ranging from 25 to $150 \mathrm{~mm} \mathrm{H}_{2} \mathrm{O}$ (six pressures). For each mitochondrion, the equilibrium radius, $R_{\mathrm{m}}$, where tongue length $L$ reached $L_{\mathrm{m}}$, was measured at the pre-set suction pressure (cf. Fig. 2f), and the aspirated mitochondrion was then released for full recovery for $5 \mathrm{~min}$ until another suction pressure was applied. Pre-tests of repeated aspirations at same pressure indicated that most of mitochondria were able to fully recover (data not shown). Membrane tension and area strain were calculated using Eqs. (1) and (2), respectively. It was found that membrane tension increased with area strain (points in Fig. 3b), which followed well with a simple linear fitting (line in Fig. 3b). The slope of fitted line represented the area compressibility modulus, ${ }^{11,15}$ which reads to be $9 \mathrm{mN} \mathrm{m}^{-1}$.

\section{Effect of Osmotic Pressure and $p H$ Value}

Membrane deformability of mitochondria is significantly affected by local physical-chemical environments where mitochondria are present. To conduct the test, osmotic pressure was varied at 170, 270, and $370 \mathrm{mOsm}$ in $\mathrm{PB}$ containing 150,250 , and $350 \mathrm{mM}$ sucrose, respectively, and $\mathrm{pH}$ value was adjusted at $6.9,7.4$, and 7.9. Dimensionless tongue length $\left(L_{\mathrm{m}} / R_{\mathrm{p}}\right)$ was used to quantify mitochondrial membrane deformability. Data indicated that the dimensionless tongue length was 




FIGURE 4. Effect of osmotic pressure and $\mathrm{pH}$ value on deformation of mitochondria suspended in PB. Plotted were the dimensionless suction lengths at $\Delta P=100 \mathrm{~mm} \mathrm{H}_{2} \mathrm{O}$ against the systematically varied sucrose concentrations of 150,250 , and $350 \mathrm{mM}$ (a), or the systematically varied $\mathrm{pH}$ values of $6.9,7.4$, and 7.9 (b). Data (bars) were presented as the mean \pm standard errors.

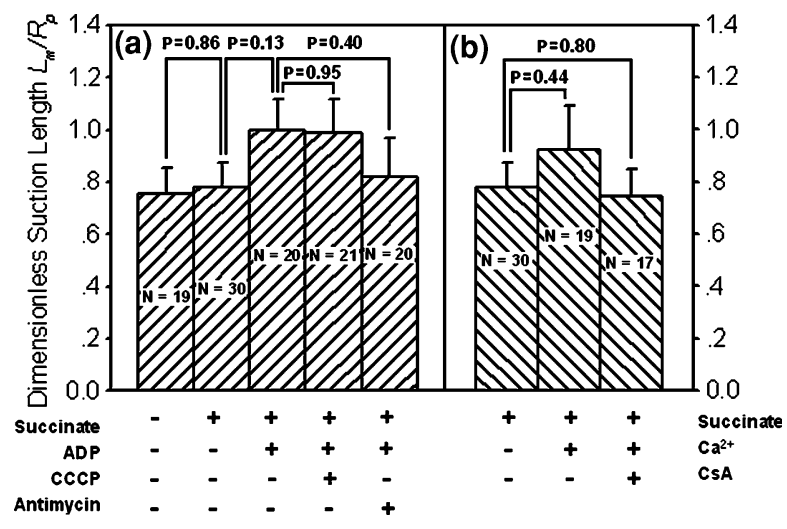

FIGURE 5. Correlation of membrane deformability with mitochondrial respiratory states or $\mathrm{Ca}^{2+}$ regulation. Plotted were the dimensionless suction lengths at $\Delta P=100 \mathrm{~mm} \mathrm{H}_{2} \mathrm{O}$ in $\mathrm{PB}, \mathrm{PB} / 10 \mathrm{mM}$ succinate, $\mathrm{PB} / 10 \mathrm{mM}$ succinate/0.5 mM ADP, $\mathrm{PB} / 10 \mathrm{mM}$ succinate/0.5 mM ADP/2.5 $\mu \mathrm{M}$ CCCP, or PB/10 mM succinate/0.5 mM ADP/10 $\mu \mathrm{M}$ antimycin (bars from left to right) (a), and in $\mathrm{PB} / 10 \mathrm{mM}$ succinate, $\mathrm{PB} / 10 \mathrm{mM}$ succinate/ $1 \mathrm{mM} \mathrm{Ca}{ }^{2+}$, or $\mathrm{PB} / 10 \mathrm{mM}$ succinate/ $1 \mathrm{mM} \mathrm{Ca}{ }^{2+} / 10 \mu \mathrm{M}$ CsA (bars from left to right) (b). Data (bars) were presented as the mean \pm standard errors.

significantly lower at $150 \mathrm{mM}$ than those at 250 and $350 \mathrm{mM}$ of sucrose concentration $\left(L_{\mathrm{m}} / R_{\mathrm{p}}=0.32 \pm\right.$ $0.07, \quad 0.78 \pm 0.09$, and $0.94 \pm 0.12$, respectively; $p<0.001$ and $p<0.001$ ) (Fig. 4a). This should be true since mitochondria were swollen at low osmotic pressure, which in turn stiffened the organelle. At higher sucrose concentrations of 250 and $350 \mathrm{mM}$, however, no significant differences in $L_{\mathrm{m}} / R_{\mathrm{p}}$ were found $(p=$ 0.27 ). By comparison, $L_{\mathrm{m}} / R_{\mathrm{p}}$ was higher at $\mathrm{pH} 7.4$ than those at $\mathrm{pH} 6.9$ and $7.9(0.78 \pm 0.09,0.52 \pm 0.08$, and $0.60 \pm 0.07$, respectively; $p=0.05$ and $p=0.13$ )
(Fig. 4b), suggesting that mitochondria were more deformable in the physiological $\mathrm{pH}$ value. It was evident that membrane deformability of mitochondria is sensitive to physical-chemical environments.

\section{Correlation of Deformability with Respiratory Functionality}

Mitochondrial functionality in cellular respiration is also correlated with their mechanical responses. ${ }^{6}$ To test this, individual mitochondria were suspended in $\mathrm{PB}$ alone (State I; inactive state), ${ }_{5}^{5} \mathrm{~PB} / 10 \mathrm{mM}$ succinate (State IV; slightly active state), ${ }^{5}$ and $\mathrm{PB} / 10 \mathrm{mM}$ succinate $/ 0.5 \mathrm{mM}$ ADP (State III; active state). ${ }^{5}$ No measurements for mitochondria suspended in $\mathrm{PB} /$ $0.5 \mathrm{mM}$ ADP (State II) were done because it is less biologically significant without the substrate. ${ }^{5}$ Data indicated that while $L_{\mathrm{m}} / R_{\mathrm{p}}$ values in States I and IV were similar $(0.75 \pm 0.10$ and $0.78 \pm 0.09$, respectively; $p=0.86)$, they were slightly lower than that in State III (1.00 $\pm 0.12 ; P=0.13)$ (three bars from the left in Fig. 5a), suggesting that mitochondria in active state be more deformable. It was evident that mitochondrial membrane deformability was, at least partly, correlated with their respiratory functionality. Mechanical responses of active mitochondria in State III, however, were unable to be abolished by respiratory chain inhibitors $\mathrm{CCCP}$ and antimycin A $(1.00 \pm 0.12,0.99 \pm 0.13$, and $0.82 \pm 0.15$, respectively; $p=0.95$ and $p=0.40$ ) (three bars from the right in Fig. 5a).

\section{Correlation of Deformability with $\mathrm{Ca}^{2+}$ Regulation}

Mechanical responses of individual mitochondria are also regulated by $\mathrm{Ca}^{2+}$, since $\mathrm{Ca}^{2+}$ is functionalized to disrupt the outer membrane and unfold the inner membrane. ${ }^{1}$ To test this, $1 \mathrm{mM} \mathrm{Ca}^{2+}$ were added into $\mathrm{PB} / 10 \mathrm{mM}$ succinate. The results demonstrated that $L_{\mathrm{m}} / R_{\mathrm{p}}$ was slightly lower, even without significant difference, for mitochondria suspended in the buffer without $\mathrm{Ca}^{2+}$ than that in the buffer supplemented with $\mathrm{Ca}^{2+}(0.78 \pm 0.09$ and $0.92 \pm 0.17$, respectively; $p=0.44$ ) (left and middle bars in Fig. 5b), indicating that disruption of outer membrane and unfolding of inner membrane by addition of $\mathrm{Ca}^{2+}$ reduced the mechanical resistances of mitochondria. As compared to mitochondria suspended in the buffer without $\mathrm{Ca}^{2+}, L_{\mathrm{m}} / R_{\mathrm{p}}$ was maintained at the baseline level $(0.74 \pm 0.11 ; p=0.80)$ when mitochondria were preincubated with immunosuppressive reagent cyclosporine A (CsA) to prevent mitochondria from $\mathrm{Ca}^{2+}$ induced disruption of outer membrane and unfolding of inner membrane (left and right bars in Fig. 5b). 

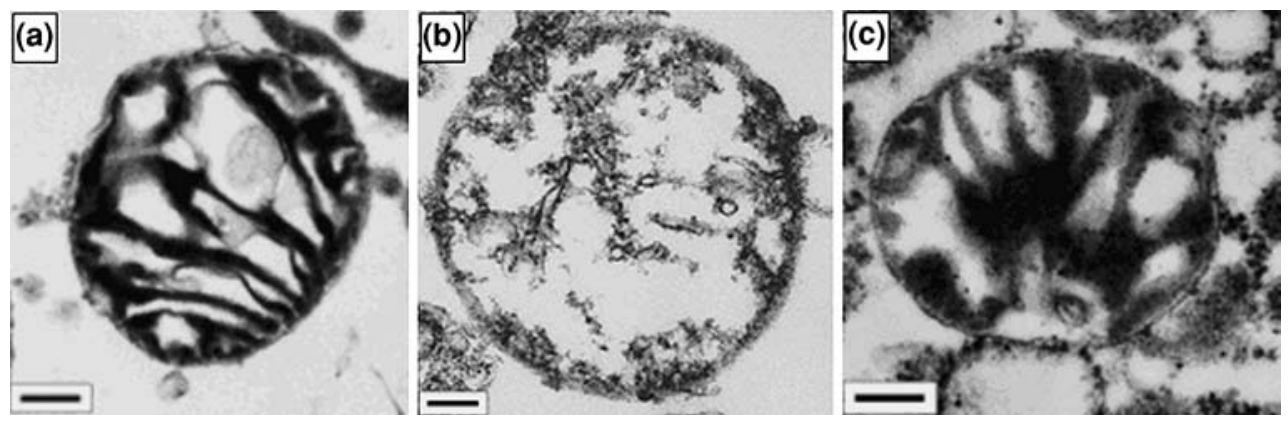

FIGURE 6. TEM images of mitochondria suspended in PB without additives (a), with the addition of $1 \mathrm{mM} \mathrm{Ca}{ }^{2+}(\mathrm{b})$, or of $1 \mathrm{mM}$ $\mathrm{Ca}^{2+} / 10 \mu \mathrm{M}$ CsA (c). Bar $=0.2 \mu \mathrm{m}$.

To further test the structural bases of the above mechanical responses, ultra-microscopic observations were performed using TEM images for mitochondria suspended in $\mathrm{PB} / 10 \mathrm{mM}$ succinate, $\mathrm{PB} / 10 \mathrm{mM}$ succinate $/ 1 \mathrm{mM} \mathrm{Ca}^{2+}$, and pre-incubated with $\mathrm{CsA}$ before adding $\mathrm{Ca}^{2+}$ in $\mathrm{PB} / 10 \mathrm{mM}$ succinate. In $\mathrm{PB} / 10 \mathrm{mM}$ succinate buffer, mitochondria had two intact layers of membranes without separation between the membranes, and the inner membrane was folded to form the cristae (Fig. 6a). When $1 \mathrm{mM} \mathrm{Ca}^{2+}$ were added, however, the integrity of outer membrane was broken down and the cristae were unfolded (Fig. 6b). Mitochondria were able to retain normal morphology when they were pre-incubated in CsA before adding $\mathrm{Ca}^{2+}$ in the buffer (Fig. 6c). Taken together, mechanical responses of individual mitochondria were apparently correlated with $\mathrm{Ca}^{2+}$ regulation, and the outer membrane was more mechanically resistant.

\section{DISCUSSIONS}

The goal of current work is to understand membrane deformability and membrane tension of individual mitochondria and to correlate the mechanical responses with their physiological functions. Isolated mitochondria had a bell-shaped size distribution with the most probable diameter of $1.0 \mu \mathrm{m}$ (Fig. 1). Aspirated deformation exhibited a transition phase where the tongue length increased with time followed by a saturation phase where it reached equilibrium (Fig. 3a). This turned to be an area compressibility modulus of $9 \mathrm{mN} \mathrm{m}^{-1}$, which was two orders-of-magnitude lower than those for red blood cells and lipid vesicles. ${ }^{11,15}$ It should also be pointed out that the membrane tension measured here was the induced tension by suction pressure rather than the one at zero area expansion. These mechanical differences may result from various composites of surface membranes and distinctive internal structures among the vesicles.
Membrane deformability and membrane tension of mitochondria were measured by micropipette aspiration assay that has been widely used for different types of cells. ${ }^{8,12,14,23}$ In a typical test, the time dependence of aspirated tongue length of a cell was obtained and the mechanic parameters were predicted by fitting the data to a mechanic model (e.g., a standard linear solid model). ${ }^{23}$ Care should be taken, however, when the assay is employed for measuring the deformation of nucleated cells or complex organelles. Mitochondria have outer and inner membrane systems, and the inner membrane folds to form the cristae which ensure to accomplish their biological functions. ${ }^{18}$ The complicated structures make it difficult to estimate directly the constitutive mechanical parameters of isolated mitochondria from a simple micropipette assay. In the current work, the maximum tongue length at a given suction pressure and the area compressibility modulus were used as apparent mechanical parameters to quantify the mechanical deformation and the surface tension of isolated mitochondria, respectively. Further investigations are required to understand the intrinsic mechanical properties of mitochondria using different mechanical models and measurements.

Membrane deformability of isolated mitochondria was affected by physical-chemical environments in buffer solution. Varying osmotic pressure or $\mathrm{pH}$ value in the buffer induced the changes in mitochondrial structures and functionality. For example, structural swelling and modified respiratory rates were found when the mitochondria were suspended in the buffer of non-physiological osmotic pressure. ${ }^{19}$ Here our data indicated that lowering the osmotic pressure reduced the mitochondrial deformation, suggesting that osmotic swelling stiffened the mitochondria. Besides, the mitochondria suspended at the physiological $\mathrm{pH}$ value were more deformable than those at the lower or higher $\mathrm{pH}$ values (Fig. 4). Regardless of mechanical variations of isolated mitochondria in different osmotic pressures and $\mathrm{pH}$ values reported here, no significant structural 
changes were found using TEM observations (data not shown).

Membrane deformability of isolated mitochondria was also correlated to mitochondrial respiration. Respiration is ceased when the substrate and ADP are absent (State I), ${ }^{20}$ and slightly activated when only the substrate succinate is present (State IV). ${ }^{5}$ This translated to the comparable deformation of isolated mitochondria. Presenting the substrate succinate and ADP induced the ADP-stimulated respiration and enhanced oxygen consumption rate (State III), which corresponded to the higher deformability. Applying CCCP (a mitochondrial uncoupler) blocked mitochondrial respiration and treatment with antimycin (an inhibitor of electron transport at complex III) caused increases in mitochondrial fragmentation, but they had no impacts on mitochondrial membrane deformability (Fig. 5a).

As an apoptotic regulator in mitochondrial physiology, $\mathrm{Ca}^{2+}$ induces the release of cytochrome $c$ and other proteins from isolated mitochondria upon a mechanism that involves increased permeability of the inner membrane, osmotic swelling of the mitochondrial matrix, and physical disruption of the outer membrane, which is so-called the membrane permeability transition (MPT). ${ }^{1,2,4,17,25}$ Such the $\mathrm{Ca}^{2+}$. induced breakage of outer membrane and unfolding of inner membrane cristae was observed for isolated rat liver mitochondria. ${ }^{1}$ This membrane disruption was also visualized in the current work when the substrate succinate and $\mathrm{Ca}^{2+}$ were presented, but was abolished when CsA (a MPT inhibitor) was pre-incubated (Figs. 6b-c). This translated to be more deformable mitochondria in the presence of $\mathrm{Ca}^{2+}$. It should be pointed out, however, that mechanical responses upon $\mathrm{Ca}^{2+}$ regulation measured using micropipette aspiration assay were not as significantly different as expected, presumably due to limited sensitivity of mechanical measurements. Thus, more sensitive mechanical measurements may be required in further investigations to elucidate the underlying mechanism.

It should also be pointed out that the mechanical behaviors of isolated mitochondria are significantly different from those of cytoplasmic mitochondria. Inside a living cell, mitochondria tend to be elongated with multi-contoured shapes, and are embedded in the dense cytoskeletal network, suggesting that mitochondrial movement, fusion and fission are restrained physically. ${ }^{13,16}$ Such the mechanical behaviors are also regulated by the cytoskeletal integrity and interactions between mitochondrial fusion/fission proteins and motor proteins. Even beyond the scope of the current study, our on-going works are focusing on understanding the cytoplasmic mitochondrial movement, fusion, and fission regulated by disrupting the cytoskeleton network, and by blocking the physical linkage between cytoskeletal proteins and motor proteins.

Finally, our results indicated that mitochondrial membrane deformation was regulated by physicalchemical environments in the buffer, and were correlated with their respiratory functions. These results provided a new insight into biomechanical bases for mechanical regulation of mitochondrial functionality.

\section{ACKNOWLEDGMENTS}

We are grateful to Center of Electron Microscopy, Institute of Biophysics, Chinese Academy of Sciences to provide TEM device, and to Lei Sun for TEM sample preparation, observation and useful discussions. This work was supported by National Natural Science Foundation of China grants 10332060 and 30730032, National Key Basic Research Foundation of China grant 2006CB910303, and Chinese Academy of Sciences grant 2005-1-16 (M.L.).

\section{REFERENCES}

${ }^{1}$ Andreyev, A., and G. Fiskum. Calcium induced release of mitochondrial cytochrome $c$ by different mechanisms selective for brain versus liver. Cell Death Differ. 6:825832, 1999.

${ }^{2}$ Armstrong, J. S. Mitochondrial membrane permeabilization. The sine qua non for cell death. Bioessays 28:253-260, 2006.

${ }^{3}$ Bandyopadhyay, S. K., and A. Dutta. Mitochondrial hepatopathies. J. Assoc. Physicians India 53:973-978, 2005.

${ }^{4}$ Brustovetsky, N., T. Brustovetsky, R. Jemmerson, and J. M. Dubinsky. Calcium-induced cytochrome $c$ release from CNS mitochondria is associated with the permeability transition and rupture of the outer membrane. J. Neurochem. 80:207-218, 2002.

${ }^{5}$ Chance, B., and G. R. Williams. Respiratory enzymes in oxidative phosphorylation III the steady state. J. Biol. Chem. 217:409-427, 1955.

${ }^{6}$ Chvanov, M. Metabolic control of elastic properties of the inner mitochondrial membrane. J. Phys. Chem. B 110:22903-22909, 2006.

${ }^{7}$ Darin, N., G. Kollberg, A.-R. Moslemi, M. Tulinius E. Holme, M. A. Grönlund, S. Andersson, and A. Oldfors. Mitochondrial myopathy with exercise intolerance and retinal dystrophy in a sporadic patient with a G583A mutation in the mt tRNA(phe) gene. Neuromuscul. Disord. 16:504-506, 2006.

${ }^{8}$ Evans, E., and A. Yeung. Apparent viscosity and cortical tension of blood granulocytes determined by micropipet aspiration. Biophys. J. 56:151-160, 1989.

${ }^{9}$ Feiguin, F., A. Ferreira, K. S. Kosik, and A. Caceres. Kinesin-mediated organelle translocation revealed by specific cellular manipulations. J. Cell Biol. 127:1021-1039, 1994. 
${ }^{10}$ Galluzzi, L., N. Larochette, N. Zamzami, and G. Kroemer. Mitochondria as therapeutic targets for cancer chemotherapy. Oncogene 25:4812-4830, 2006.

${ }^{11}$ Kwok, R., and E. Evans. Thermoelasticity of large lecithin bilayer vesicles. Biophys. J. 35:637-652, 1981.

${ }^{12}$ Long, M., Z. Wu, H. Wang, G. Song, X. Wang, and Y. Wu. Experimental investigation on viscoelasticity of hepatocytes. Acta Bioph. Sin. 12:169-173, 1996.

${ }^{13}$ Miller, K. E., and M. P. Sheetz. Axonal mitochondrial transport and potential are correlated. J. Cell Sci. 117:2791-2804, 2004.

${ }^{14}$ Needham, D., and R. M. Hochmuth. A sensitive measure of surface stress in the resting neutrophil. Biophys. J. 61:1664-1670, 1992.

${ }^{15}$ Nichols-Smith, S., S. The, and T. L. Kuhl. Thermodynamic and mechanical properties of model mitochondrial membranes. Biochim. Biophys. Acta. 1663:82-88, 2004.

${ }^{16}$ Penman, S. Rethinking cell structure. Proc. Natl. Acad. Sci. USA 92:5251-5257, 1995.

${ }^{17}$ Petit, P. X., M. Goubern, P. Diolez, S. A. Susin, N. Zamzami, and G. Kroemer. Disruption of the outer mitochondrial membrane as a result of large amplitude swelling: the impact of irreversible permeability transition. FEBS Lett. 426:111-116, 1998.

${ }^{18}$ Scheffler, I. E. Mitochondria make a come back. Adv. Drug Deliv. Rev. 49:3-26, 2001.
${ }^{19}$ Sitaramam, V., D. Sambasivarao, and J. C. Mathai. Differential effects of osmotic pressure on mitochondrial respiratory chain and indices of oxidative phosphorylation. Biochim. Biophys. Acta. 975:252-266, 1989.

${ }^{20}$ Toleikis, A., S. Trumbeckaite, and D. Majiene. Cytochrome $c$ effect on respiration of heart mitochondria: influence of various factors. Biosci. Rep. 25:387-397, 2005.

${ }^{21}$ Varadi, A., L. I. Johnson-Cadwell, V. Cirulli, Y. Yoon V. J. Allan, and G. A. Rutter. Cytoplasmic dynein regulates the subcellular distribution of mitochondria by controlling the recruitment of the fission factor dynaminrelated protein-1. J. Cell Sci. 117:4389-4400, 2004.

${ }^{22}$ Wagner, O. I., J. Lifshitz, P. A. Janmey, M. Linden, T. K. McIntosh, and J.-F. Leterrier. Mechanisms of mitochondria-neurofilament interactions. J. Neurosci. 23:9046-9058, 2003.

${ }^{23} \mathrm{Wu}$, Z., G. Zhang, M. Long, H. Wang, G. Song, and $\mathrm{S}$. Cai. Comparison of the viscoelastic properties of normal hepatocytes and hepatocellular carcinoma cells under cytoskeletal perturbation. Biorheology 37:279-290, 2000.

${ }^{24}$ Yaffe, M. P. The machinery of mitochondrial inheritance and behavior. Science 283:1493-1497, 1999.

${ }^{25}$ Yi, M., D. Weaver, and G. Hajnóczky. Control of mitochondrial motility and distribution by the calcium signal: a homeostatic circuit. J. Cell Biol. 167:661-672, 2004. 